\title{
ANOTHER PROOF OF HUREWICZ THEOREM
}

\author{
MiroslaV REPICKÝ
}

\begin{abstract}
A Hurewicz theorem says that every coanalytic non- $G_{\delta}$ set $C$ in a Polish space contains a countable set $Q$ without isolated points such that $\bar{Q} \cap C=Q$. We present another elementary proof of this theorem and generalize it for $\kappa$-Suslin sets. As a consequence, under Martin's Axiom, we obtain a characterization of $\boldsymbol{\Sigma}_{2}^{1}$ sets that are the unions of less than the continuum closed sets.
\end{abstract}

\section{Hurewicz schemes}

Several proofs of the Hurewicz theorem are known. The original proof by W. Hurewicz [2] is based on the notion of "Häufungsystem" (which we call Hurewicz scheme below). The proof presented by A. Kechris [3, Theorem 21.18 and Theorem 21.22] is based on the so called separation game. Recently Michal Sta š 5 gave another simple and elementary proof of this theorem. Our proof is relative to the proof by M. Staš but, in addition, we introduce a notion of a $D$-proper mapping between metric spaces and obtain another variant of his characterization. We present a natural generalization to $\kappa$-Suslin sets.

Definition 1.1. Let $X$ be a metric space.

(1) A mapping $\varphi$ : ${ }^{<\omega} \omega \rightarrow X$ is an $H$-scheme on $X$ (i.e., Hurewicz scheme) if the following two conditions are satisfied:

(a) $\varphi\left(s^{\frown} n\right) \neq \varphi\left(s^{\frown} m\right)$ for all $s \in{ }^{<\omega} \omega$ and $n \neq m$,

(b) $\varphi(s)=\lim _{n \rightarrow \infty} \varphi\left(s^{\frown} n\right)$ for all $s \in<\omega \omega$.

(2) An $H$-scheme $\varphi$ is separated if there is a system of open sets $\left\{V_{s}: s \in{ }^{<\omega} \omega\right\}$ such that for all $s \in{ }^{<\omega} \omega$,

(c) $\varphi(s) \in V_{s} \backslash \bigcup_{n \in \omega} \overline{V_{s \frown n}}$, and

(d) $\left\{\overline{V_{s \frown n}}: n \in \omega\right\}$ is a disjoint system of subsets of $V_{s}$.

(C) 2011 Mathematical Institute, Slovak Academy of Sciences.

2010 Mathematics Subject Classification: Primary 03E15; Secondary 03E17, 03E50.

Keywords: Hurewicz scheme, $D$-proper mapping, analytic set.

The work has been supported by grant of Slovak Grant Agency VEGA 1/0032/09. 


\section{MIROSLAV REPICKÝ}

We say that the system $\left\{V_{s}: s \in{ }^{<\omega} \omega\right\}$ is normal if

(e) $\lim _{s \in<\omega \omega} \operatorname{diam}\left(V_{s}\right)=0$, i.e., $(\forall \varepsilon>0)\left(\forall^{\infty} s \in<\omega \omega\right) \operatorname{diam}\left(V_{s}\right)<\varepsilon$.

(3) An $H$-scheme $\varphi:{ }^{<\omega} \omega \rightarrow X$ is complete (in $X$ ), if for every $g \in{ }^{\omega} \omega$, the sequence $\left\{\varphi(g\lceil n)\}_{n \in \omega}\right.$ converges in $X$.

Definition 1.2. For an $H$-scheme $\varphi:{ }^{<\omega} \omega \rightarrow X$ we denote

$$
\begin{aligned}
& H(\varphi)=\overline{\operatorname{rng}(\varphi)} \backslash \operatorname{rng}(\varphi), \\
& G(\varphi)=\left\{x \in X:\left(\exists f \in{ }^{\omega} \omega\right) x=\lim _{n \rightarrow \infty} \varphi(f\lceil n)\} .\right.
\end{aligned}
$$

It is easy to see that $H(\varphi)$ is a $G_{\delta}$ set and, if $X$ is a Polish space, then $G(\varphi)$ is analytic. For every $H$-scheme $\varphi$ we can define a (partial) function $f_{\varphi}:{ }^{\omega} \omega \rightarrow X$ by $f_{\varphi}(x)=\lim _{n \rightarrow \infty} \varphi\left(x\lceil n)\right.$, if the limit exists. The set $G(\varphi)$ is the range of $f_{\varphi}$. The function $f_{\varphi}$ is total if and only if $\varphi$ is a complete $H$-scheme. If $\varphi$ is an $H$-scheme on a complete metric space separated by a normal system of open sets, then $f_{\varphi}$ is total, injective, and continuous.

By $\mathrm{H} \mathrm{ur}$ e w i c z [2], an $H$-scheme $\varphi$ is normal, if it satisfies condition $\overline{\operatorname{rng}(\varphi)}=$ $G(\varphi) \cup \operatorname{rng}(\varphi)$, i.e., $H(\varphi) \subseteq G(\varphi)$. We need a stronger property because of the next lemma.

Lemma 1.3. Let $\varphi:{ }^{<\omega} \omega \rightarrow X$ be an $H$-scheme on a metric space $X$ separated by a normal system of open sets $\left\{V_{s}: s \in{ }^{<\omega} \omega\right\}$. If $\left\langle s_{n}: n \in \omega\right\rangle$ is a sequence in $<\omega \omega, T=\left\{s \in{ }^{<\omega} \omega: \exists^{\infty} n s \subseteq s_{n}\right\}$, and $x \in X$, then $x=\lim _{n \rightarrow \infty} \varphi\left(s_{n}\right)$ if and only if one of the following conditions holds:

(i) there is $s \in{ }^{<\omega} \omega$ such that $T=\{s|k: k \leq| s \mid\}, \forall \forall^{\infty} n \subseteq s_{n}$, and $x=\varphi(s)$, or

(ii) there is $g \in{ }^{\omega} \omega$ such that $T=\left\{g\lceil k: k \in \omega\}, \forall k \forall \forall^{\infty} n g\left\lceil k \subseteq s_{n}\right.\right.$, and $x=\lim _{n \rightarrow \infty} \varphi(g\lceil n) \notin \operatorname{rng}(\varphi)$.

In particular, $G(\varphi)=H(\varphi)$.

Proof. Assume that $x=\lim _{n \rightarrow \infty} \varphi\left(s_{n}\right)$. For every $s \in T$ we have $x \in \overline{V_{s}}$. By condition (d) it follows that $T$ does not contain any two incomparable elements. If $T$ is finite, let $s$ be its maximal element. There are increasing sequences $\left\{n_{k}\right\}_{k \in \omega}$ and $\left\{m_{k}\right\}_{k \in \omega}$ such that $s \frown m_{k} \subseteq s_{n_{k}}$ for all $k$. Then

$$
d\left(\varphi(s), \varphi\left(s_{n_{k}}\right)\right) \leq d\left(\varphi(s), \varphi\left(s \frown m_{k}\right)\right)+\operatorname{diam}\left(V_{s \frown m_{k}}\right)
$$

and hence

$$
\varphi(s)=\lim _{k \rightarrow \infty} \varphi\left(s_{n_{k}}\right)=x .
$$

If $t$ is a proper subsequence of $s$, then $\varphi(t) \in V_{t} \backslash \overline{V_{s}}$ and so $\varphi(t) \neq x$. Hence we cannot apply the same argument for any $t$ below $s$ which means that $\forall^{\infty} n$ $s \subseteq s_{n}$. 


\section{ANOTHER PROOF OF HUREWICZ THEOREM}

If $T$ is infinite, then there must be $g \in{ }^{\omega} \omega$ such that $T=\{g\lceil k: k \in \omega\}$. Then for every $k, \varphi\left(g\lceil k) \in V_{g \nmid k} \backslash \overline{V_{g \uparrow(k+1)}}\right.$, hence $\varphi(g\lceil k) \neq x$ and, like in the previous case, $\forall k \forall^{\infty} n g\left\lceil k \subseteq s_{n}\right.$. Then $x=\lim _{n \rightarrow \infty} \varphi\left(g\lceil n)\right.$ because $x \in \bigcap_{n \in \omega} \overline{V_{g\lceil n}}$.

EXAmple 1.4. The Cantor space ${ }^{\omega} 2$ can be represented as a topological closure of an $H$-scheme separated by a normal system of open sets. By induction let us define $t_{s} \in{ }^{<\omega} 2$ for $s \in{ }^{<\omega} \omega: t_{\emptyset}=\emptyset$ and $t_{s} \frown n=t_{s} \frown 0^{n} 1$. Then $\varphi_{C}:{ }^{<\omega} \omega \rightarrow{ }^{\omega} 2$ defined by $\varphi_{C}(s)=t_{s} \frown \overline{0}(\overline{0}$ is the infinite sequence of 0 's $)$ is an $H$-scheme separated by the normal system of clopen sets $\left\{\left[t_{s}\right]: s \in<\omega \omega\right\}$. Clearly, $\overline{\operatorname{rng}\left(\varphi_{C}\right)}={ }^{\omega} 2$ and $G\left(\varphi_{C}\right)=H\left(\varphi_{C}\right)={ }^{\omega} 2 \backslash \operatorname{rng}\left(\varphi_{C}\right) \simeq{ }^{\omega} \omega$.

TheOREM 1.5. If $X$ is a metric space and $\varphi:{ }^{<\omega} \omega \rightarrow X$ is a complete $H$-scheme separated by a normal system of open sets, then $H(\varphi)=G(\varphi) \simeq{ }^{\omega} \omega$ and $\overline{\operatorname{rng}(\varphi)}$ is a compact perfect set homeomorphic to the Cantor space.

Proof. Let $\psi:{ }^{<\omega} \omega \rightarrow Y$ be any other complete $H$-scheme on a metric space $Y$ separated by a normal system of open sets. There is a homeomorphism $f: \overline{\operatorname{rng}(\varphi)} \rightarrow \overline{\operatorname{rng}(\psi)}$ such that $f(\varphi(s))=\psi(s)$ and $f\left(f_{\varphi}(g)\right)=f_{\psi}(g)$ for all $s \in<\omega_{\omega}$ and $g \in \omega^{\omega} \omega$. This is possible because the convergence of $\left\{\varphi\left(s_{n}\right)\right\}_{n \in \omega}$ and $\left\{\psi\left(s_{n}\right)\right\}_{n \in \omega}$ depends on the sequence $\left\{s_{n}\right\}_{n \in \omega}$ in the way described by Lemma 1.3. Apply this fact to the $H$-scheme on the Cantor space from Example 1.4.

\section{Hurewicz theorem}

Definition 2.1. Let $X$ be a metric space. An $H$-scheme $\varphi^{\prime}:{ }^{<\omega} \omega \rightarrow X$ is a subscheme of an $H$-scheme $\varphi:{ }^{<\omega} \omega \rightarrow X$, if there is an injective mapping $h:{ }^{<\omega} \omega \rightarrow{ }^{<\omega} \omega$ such that $|h(s)|=|s|, t \subseteq s$ implies $h(t) \subseteq h(s)$, and $\varphi^{\prime}(s)=$ $\varphi(h(s))$ for all $s \in<\omega \omega$.

Subschemes of $H$-schemes preserve normality, separateness, and completeness. If $\varphi^{\prime}$ is a subscheme of an $H$-scheme $\varphi$, then $\operatorname{rng}\left(\varphi^{\prime}\right) \subseteq \operatorname{rng}(\varphi)$ and $G\left(\varphi^{\prime}\right) \subseteq G(\varphi)$. The notion of a subscheme is similar to the notion of "Restsystem" in [2].

LeMma 2.2. Every $H$-scheme has a subscheme separated by a normal system of open sets.

Proof. We define open balls $V_{s}$ and a function $h:<\omega_{\omega} \rightarrow{ }^{<\omega} \omega$ by induction on $|s|$ for $s \in{ }^{<\omega} \omega$ so that

$$
\varphi(h(s)) \in V_{s} \quad \text { and } \quad \operatorname{diam}\left(V_{s}\right)<2^{-(n+s(n))} \quad \text { for } \quad s \in{ }^{n+1} \omega .
$$




\section{MIROSLAV REPICKÝ}

Set $h(\emptyset)=\emptyset$ and let $V_{\emptyset}$ be an open ball with center $\varphi(h(\emptyset))$. Let $s \in<\omega_{\omega}$ be arbitrary and let us assume that $h(s)$ and $V_{s}$ are defined and $\varphi(h(s)) \in V_{s}$. Let $\left\{k_{n}^{s}\right\}_{n \in \omega}$ be an increasing sequence such that

$\varphi\left(h(s) \frown k_{n}^{s}\right) \in V_{s} \quad$ and $\quad d\left(\varphi\left(h(s) \frown k_{n+1}^{s}\right), \varphi(h(s))\right)<d\left(\varphi\left(h(s) \frown k_{n}^{s}\right), \varphi(h(s))\right)$.

Set $h(s \frown n)=h(s) \frown k_{n}^{s}$ and choose open balls $V_{s \frown n} \subseteq V_{s} \backslash\{\varphi(s)\}$ with centers $\varphi(h(s \frown n))$ for $n \in \omega$ so that their closures are pairwise disjoint, do not contain $\varphi(h(s))$, and their diameters are sufficiently small.

Definition 2.3. Let $f: Y \rightarrow X$ and $f(Y) \subseteq D \subseteq X$. The function $f$ is said to be $D$-proper, if for every nonempty open set $U \subseteq Y$ the set $\overline{f(U)} \backslash D$ is nonempty and has no isolated points.

Lemma 2.4. Let $X$ and $Y$ be metric spaces, let $f: Y \rightarrow X$ be continuous, and let $f(Y) \subseteq D \subseteq X$. If $Y$ is complete and $f$ is D-proper, then there exists a complete $H$-scheme $\varphi:{ }^{<\omega} \omega \rightarrow X \backslash D$ such that $G(\varphi) \subseteq f(Y)$.

Proof. By induction on $|s|$ for $s \in{ }^{<\omega} \omega$ we define a sequence $\left\langle V_{s}: s \in{ }^{<\omega} \omega\right\rangle$ of nonempty open balls in $Y$ and an $H$-scheme $\varphi:{ }^{<\omega} \omega \rightarrow X \backslash D$ such that

(1) $\operatorname{diam}\left(V_{s}\right)<2^{-|s|}$ and $\operatorname{diam}\left(\overline{f\left(V_{s}\right)}\right)<2^{-|s|}$,

(2) $\left\{\overline{V_{s \frown n}}: n \in \omega\right\}$ is a disjoint system of subsets of $V_{s}$,

(3) $\varphi(s) \in \overline{f\left(V_{s}\right)} \backslash D$.

Let $V_{\emptyset} \subseteq Y$ be any open ball such that $\operatorname{diam}\left(V_{\emptyset}\right)<1$ and $\operatorname{diam}\left(\overline{f\left(V_{\emptyset}\right)}\right)<1$, and let $\varphi(\emptyset) \in \overline{f\left(V_{\emptyset}\right)} \backslash D$. This is possible because $f$ is continuous and $D$-proper.

Let us assume that $V_{s}$ and $\varphi(s) \in \overline{f\left(V_{s}\right)} \backslash D$ have been constructed for a given $s \in{ }^{m} \omega$. Since $\varphi(s)$ is not an isolated point of $\overline{f\left(V_{s}\right)} \backslash D$, we can choose a disjoint sequence $\left\langle U_{n}: n \in \omega\right\rangle$ of open balls in $X$ such that $U_{n} \cap\left(\overline{f\left(V_{s}\right)} \backslash D\right) \neq \emptyset$, $U_{n} \subseteq B\left(\varphi(s), 2^{-m-1}\right)$, and $\varphi(s) \notin \overline{U_{n}}$; hence $\operatorname{diam}\left(U_{n}\right)<2^{-m-1}$. For each $n \in \omega$ let us choose an open ball $V_{s} \frown n \subseteq f^{-1}\left(U_{n}\right) \cap V_{s}$ in $Y$ with diameter $<2^{-m-1}$ such that $\overline{f\left(V_{s \frown n}\right)} \subseteq U_{n}$ and let $\varphi(s \frown n) \in \overline{f\left(V_{s \frown n}\right)} \backslash D$.

Clearly, $\varphi$ is an $H$-scheme and conditions (1)-(3) are satisfied. We prove that $G(\varphi) \subseteq f(Y)$. Let $g \in{ }^{\omega} \omega$. Since $Y$ is complete there is a unique $y \in \bigcap_{m \in \omega} \overline{V_{g \nmid m}}$. Then $f(y)=\lim _{m \rightarrow \infty} \varphi\left(g\lceil m)\right.$ because $f(y) \in f\left(\overline{V_{g \nmid m}}\right) \subseteq \overline{f\left(V_{g\lceil m}\right)}$ and hence $d\left(f(y), \varphi(g\lceil m))<2^{-m}\right.$.

Let $\kappa$ be an infinite cardinal number. For a set $D \subseteq X$ let $I_{<\kappa}(D)$ denote the ideal over $D$ generated by unions of $<\kappa$ closed sets in $X$ which are subsets of $D$ and let $I_{\kappa}(D)=I_{<\kappa^{+}}(D)$. Let us recall that $\operatorname{cov}(\mathcal{M})$ denotes the least cardinality of a family of meager sets covering the real line. 


\section{ANOTHER PROOF OF HUREWICZ THEOREM}

Theorem 2.5. Let $\omega \leq \kappa<\operatorname{cov}(\mathcal{M})$. Let $X$ be a metric space, let $A \subseteq D \subseteq X$, and let $A$ be a $\kappa$-Suslin set. The following conditions are equivalent:

(1) $A \notin I_{<\operatorname{cov}(\mathcal{M})}(D)$.

(2) $A \notin I_{\kappa}(D)$.

(3) There is a continuous D-proper mapping $f: Y \rightarrow X$ for some complete metric space $Y$ such that $f(Y) \subseteq A$ and the weight of $Y$ is less or equal to $\kappa$.

(4) There is a continuous D-proper mapping $f: Y \rightarrow X$ for some complete metric space $Y$ such that $f(Y) \subseteq A$.

(5) There is a complete $H$-scheme $\varphi:{ }^{<\omega} \omega \rightarrow X \backslash D$ such that $G(\varphi) \subseteq A$.

(6) There is a complete $H$-scheme $\varphi:{ }^{<\omega} \omega \rightarrow X \backslash D$ separated by a normal system of open sets such that $G(\varphi) \subseteq A$.

(7) There is a compact perfect set $P \subseteq X$ such that $P \cap D \subseteq A, P \backslash D$ is countable, and $\overline{P \backslash D}=P$.

Pr o of. The implications $(1) \Rightarrow(2)$ and $(3) \Rightarrow(4)$ are trivial, the implication $(4) \Rightarrow(5)$ is Lemma 2.4 and $(5) \Rightarrow(6)$ follows by Lemma 2.2 ,

$(2) \Rightarrow(3)$ Let $g:{ }^{\omega} \kappa \rightarrow X$ be a continuous function such that $g\left({ }^{\omega} \kappa\right)=A$. As ${ }^{\omega} \kappa$ has a base of open sets of size $\kappa$, there is a maximal open set $U_{0}$ in ${ }^{\omega_{\kappa}}$ such that $g\left(U_{0}\right) \in I_{\kappa}(D)$. Denote $Y={ }^{\omega} \kappa \backslash U_{0}$ and $f=g \uparrow Y$. The function $f: Y \rightarrow X$ is continuous, $Y$ is a complete metric space, and $f(Y) \subseteq A$. We verify that $f$ is $D$-proper.

Let $V$ be an open set in $Y$, i.e., $V=U \backslash U_{0}$ for some open set $U \subseteq \omega_{\kappa}$. If $\overline{f(V)} \subseteq D$, then $g(U)=\left(g(U) \backslash g\left(U_{0}\right)\right) \cup\left(g(U) \cap g\left(U_{0}\right)\right) \subseteq g(V) \cup g\left(U_{0}\right) \subseteq$ $\overline{f(V)} \cup g\left(U_{0}\right) \in I_{\kappa}(D)$ and hence $V \subseteq U \subseteq U_{0}$. It follows that if $V$ is a nonempty open set in $Y$, then $\overline{f(V)} \backslash D \neq \emptyset$. We prove that $\overline{f(V)} \backslash D$ has no isolated points. On the contrary, assume that $x \in \overline{f(V)} \backslash D$ is an isolated point and $B$ is an open ball in $X$ such that $B \cap \overline{f(V)} \backslash D=\{x\}$. The set $F=\overline{f(V)} \cap B \backslash\{x\}$ is an $F_{\sigma}$ set in $X$ contained in $D$, the set $V^{\prime}=V \cap f^{-1}(B \backslash\{x\})$ is open in $Y$, and $g\left(V^{\prime}\right) \subseteq f(V) \cap B \backslash\{x\} \subseteq F \in I_{\kappa}(D)$. Let $U^{\prime} \subseteq \omega_{\kappa}$ be such an open set that

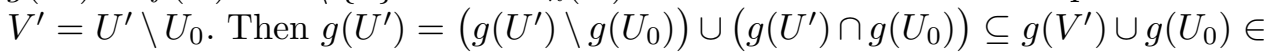
$I_{\kappa}(D)$. It follows that $V^{\prime}=\emptyset$ because $V^{\prime} \subseteq U^{\prime} \subseteq U_{0}$. Then $f(V) \cap B \backslash\{x\}=\emptyset$ and $x \in f(V) \subseteq D$ because $x \in \overline{f(V)}$. This is a contradiction.

$(6) \Rightarrow(7)$ If $\varphi:{ }^{<\omega} \omega \rightarrow X \backslash D$ is a complete $H$-scheme separated by a normal system of open sets, then by Theorem 1.5, $P=\overline{\operatorname{rng}(\varphi)}$ is a compact perfect set, and since $G(\varphi)=H(\varphi) \subseteq A \subseteq D$, then $P \cap D=G(\varphi)$ and $P \backslash D=\operatorname{rng}(\varphi)$.

$(7) \Rightarrow(1)$ Assume that $P$ is a compact perfect set satisfying (7). Then $P \backslash A=$ $P \backslash D$ is a countable dense subset of $P$. So, if $A \in I_{<\operatorname{cov}(\mathcal{M})}(D)$, then $P \cap A$ and 


\section{MIROSLAV REPICKÝ}

hence also $P$ is the union of $<\operatorname{cov}(\mathcal{M})$ nowhere dense subsets of $P$. This is impossible and therefore $A \notin I_{<\operatorname{cov}(\mathcal{M})}(D)$.

Let us note that the proof of the theorem requires neither that the metric space $X$ is complete nor that $X$ has a countable base. If we use another definition of a $\kappa$-Suslin set, then there may be some restrictions (for example, a set in a metric space of weight $\leq \kappa$ is a result of the Suslin operation $\mathcal{A}^{\kappa}$ applied to closed sets if and only if it is a continuous image of $\omega_{\kappa}$, see [4, Proof of Theorem 2B.1]).

Condition (3) as a variant of (4) was suggested by the referee.

Michal Staš [5] has proved the equivalence of conditions closely related to $(2) \Leftrightarrow(6) \Leftrightarrow(7)$ for analytic sets (i.e., $\omega_{1}$-Suslin sets). His proof of the implication $(2) \Rightarrow(6)$ contains a direct construction of an $H$-scheme by induction using the following lemma:

Lemma 2.6. Let $X$ be a space with a countable base of open sets. Let $A \subseteq D \subseteq X$. Then for every open set $U \subseteq X$ such that $A \cap U \notin I_{\omega}(D)$ there are infinitely many points $p \in U \backslash D$ such that $A \cap V \notin I_{\omega}(D)$ for all neighbourhoods $V$ of $p$.

Let us look at the meaning of the clause "infinitely many points $p \in U$ " in the lemma. Let us note that the set $A$ in the lemma need not be analytic. But if $A$ is analytic and $A \cap U \notin I_{\omega}(D)$, then by Theorem 2.5 there is a continuous $D$-proper mapping $f: Y \rightarrow X$ defined on a Polish space $Y$ such that $f(Y) \subseteq A \cap U$. For every open set $V \subseteq X$ such that $f(Y) \cap V \neq \emptyset$ the function $f \uparrow\left(f^{-1}(V)\right)$ is again $D$-proper. Hence for every $p \in \overline{f(Y)} \backslash D$ and for every neighbourhood $V$ of $p$ we have $A \cap V \notin I_{\omega}(D)$.

It is well-known that every $\boldsymbol{\Sigma}_{2}^{1}$ set is $\omega_{1}$-Suslin. By Theorem 2.5, if $\operatorname{cov}(\mathcal{M})>\omega_{1}$, then a $\Sigma_{2}^{1}$ set $A$ in a (possibly non-complete) metric space is the union of $\leq \omega_{1}$ closed sets if and only if there is no compact perfect set $P$ such that $P \backslash A$ is countable dense in $P$. (Take $D=A$ in the theorem.) In particular, we have:

Corollary 2.7. If Martin's Axiom holds, then a $\boldsymbol{\Sigma}_{2}^{1}$ set $A$ is the union of $<2^{\omega}$ closed sets if and only if there is no compact perfect set $P$ such that $P \backslash A$ is countable dense in $P$.

\section{REFERENCES}

[1] BARTOSZYŃSKI, T.-JUDAH, H.: Set Theory. On the Structure of the Real Line. A. K. Peters, Ltd., Wellesley, MA, 1995.

[2] HUREWICZ, W.: Relativ perfekte Teile von Punktmengen und Mengen (A), Fund. Math. 12 (1928), 78-109. 


\section{ANOTHER PROOF OF HUREWICZ THEOREM}

[3] KECHRIS, A. S.: Classical Descriptive Set Theory. Springer-Verlag, New York, 1994.

[4] MOSCHOVAKIS, Y. N.: Descriptive Set Theory, in: Stud. Logic Found. Math., Vol. 100, North-Holland Publ. Comp., Amsterdam, 1980.

[5] STAŠ, M.: Hurewicz scheme, Acta Univ. Carolin. Math. Phys. 49 (2008), 75-78.

Received February 25, 2010

Mathematical Institute

Slovak Academy of Sciences

Jesenná 5

SK-041-54 Košice

SLOVAKIA

Department of Computer Science Faculty of Science

P. J. Šafárik University

Jesenná 5

SK-041-54 Košice

SLOVAKIA

E-mail: repicky@kosice.upjs.sk 